\title{
The radial extent of the Fornax cluster Low Surface Brightness galaxy population
}

\author{
J. I. Davies, A. Kambas, Z. Morshidi-Esslinger and R. Smith \\ Department of Physics and Astronomy, University of Cardiff, Cardiff, \\ $U K$
}

\begin{abstract}
In this paper we compare the radial distribution of Fornax cluster galaxies selected at different surface brightness values. The bright galaxy ( $\mathrm{RC} 3$ ) sample surface brightness distribution peaks at a $\mu_{x}^{B}=21.5$, the Low Surface Brightness (LSB) sample at $\mu_{x}^{B}=23.7$ and the very Low Surface Brigthness (VLSB) sample at $\mu_{x}^{B}=25.7$. The bright galaxy surface density decreases with an exponential scale length of $\alpha=0.5^{\circ}$, the LSB galaxies with $\alpha=1.3^{\circ}$ and the VLSB galaxies with $\alpha=2.0^{\circ}$. Thus the LSB and VLSB populations define a mass scale size some 2.5 and 4 times larger than the bright galaxies respectively. The contribution of the VLSB population to the total cluster luminosity is comparible to that of the brighter galaxies. If their mass to light ratios are significantly larger than than that of the bright galaxies they will dominate the mass and possibly account for the missing mass in clusters.
\end{abstract}

\section{Introduction}

An important issue in observational cosmology is the characteristic scales over which mass is distributed. Recently there has been a surge in interest in trying to relate the locally observed mass distribution to that determined from the fluctuations in the microwave background. The approach in the main has been to use numerical models to simulate the expected distribution of galaxies given the early distribution of matter defined by the CMB fluctuations. From this one can try to infer the dominant physical processes at work and the important cosmological parameters. The interpretation of these simulations rely on measurements of the local mass distribution determined from observations of relatively nearby galaxies. In many cases a biasing parameter is used to relate the models to observations because the bright galaxies are thought to be "biased" tracers of the mass, in the sense that they trace out smaller scale structure than the underlying "true" distribution of mass (see Jenkins et al., (1998). In this paper we ask the question "are LSB galaxies more accurate tracers of the underlying mass distribution in the Universe ?". We have identified three samples of Fornax cluster galaxies selected by their surface brightness and we compare the length scales over which they are distributed. We also assess their contribution to the total luminosity (mass ?) of the cluster. 


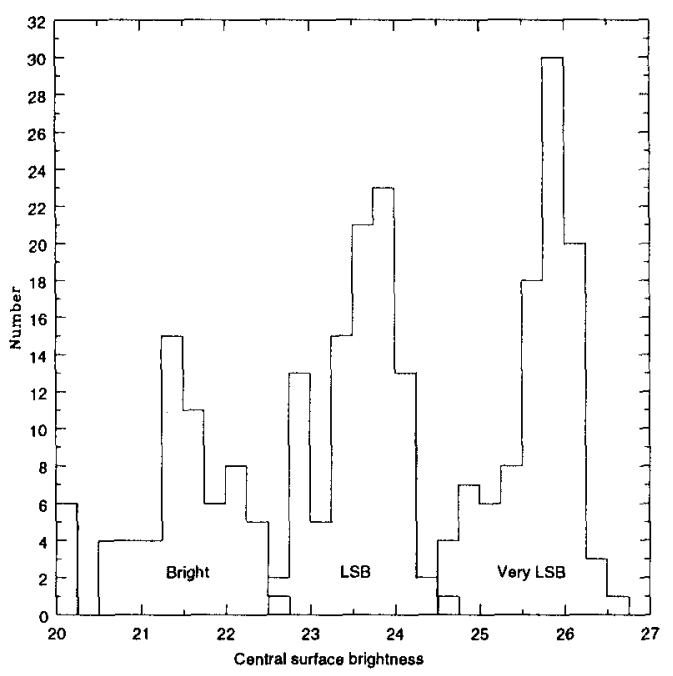

Figure 1. The B band surface brightness distributions of the three samples. Numbers in the VLSB sample have been scaled (reduced) by a factor of 20 so that all the samples can be easily displayed on the same plot.

\section{The bright galaxy sample}

We have taken the bright galaxy sample from the paper by Jones and Jones (1980). They list 64 Fornax cluster galaxies with $\mathrm{m}_{B} \leq 16$. We have previously measured the surface brightness distribution of these galaxies (Disney et al. 1990) and we reproduce this in fig 1. This sample illustrates the familiar Freeman result. The distribution of surface brightness is sharply peaked at a value of $\approx 21.5 \mathrm{~B} \mu$ with an rms width of $\approx 0.4 \mathrm{~B} \mu$. These galaxies will be used to define the mass distribution as delineated by the bright galaxies.

\section{The LSB sample}

For this sample we use data from an APM scan of UKST photographic plate (f358). The APM machine scans an area of $5.8^{\circ} \times 5.8^{\circ}$ of a $6^{\circ} \times 6^{\circ}$ UKSTU photographic plate following a 2 pass procedure. First, it determines the sky background as a function of position on the plate. Secondly, it detects images which are in the form of connected pixels above a predetermined threshold above sky.

In addition, the processing computer assigns a flag number to each image it picks up. This is done by looking at the distribution of various image parameters of all detected objects; stars, galaxies, plate defects, other noise, etc. (Maddox 1988). A star which falls along a well defined stellar ridge line is tagged as -1 . Other objects are plotted in the same manner and their separations from 
the stellar ridge line are measured. Images which are more than $2.0 \sigma$ away are tagged with 1 as non-stellar objects (galaxies). The remaining objects with parameters which are highly unlikely for real stars and galaxies are tagged with 0 as noise. Each image is also assigned 16 scan parameters, these include the isophotal intensity, position and the image area at 8 isophotal levels (the 'areal' profile).

Only objects which are classified by the APM as galaxies are included in our sample. All of the objects we are interested in are assumed to have exponential light profiles (Davies, 1989), so we have fitted the APM areal profiles to an exponential (i.e. $I=I_{0} e^{-\left(\frac{r}{\alpha}\right)}$ ) and calculated $\mu_{0}, \alpha$ and $m_{B}$.

The calibration of the Fornax Plate was carried out using data from Davies et al. (1988). The detection isophote was set to $\mu_{L}^{B}=25.6$. We made several searches for LSB galaxies in the Fornax Cluster and adjusted our selection criteria to optimise our detection technique, i.e. to minimise the number of background contaminating galaxies and to maximise the number of detected cluster LSB galaxies. Checking against Ferguson (1989) and the Digitized Sky Survey, test samples of Fornax galaxies with scale lengths smaller than 3.0 arcseconds or with central surface brightnesses brighter than $22.5 \mathrm{~B} \mathrm{mag} / \mathrm{arcsec}^{2}$ suffer greater background contamination and larger numbers of spurious detections (see also Scwartzenberg 1996). Based on the results of our trial runs, the search technique was optimised using the following selection criteria: $\mu_{0}$ fainter than 22.5 $\mathrm{B} \mathrm{mag} / \operatorname{arcsec}^{2}$ and $\alpha$ larger than 3.0 arcseconds.

Checking each object we detected on the plate which satisfy the selection criteria against the Digitized Sky Survey we found that $94 \%$ of the detected objects were genuine LSB galaxies while the remaining objects were either stellar halos, edges of bright galaxies or plate defects. Having removed the spurious detections from our test sample we compared the results with those of Davies et al. (1988), Caldwell (1987) and Ferguson (1989) and Irwin et al. 1990.

Using a cross-reference against Ferguson (1989) we found that 70 of our detections were listed as cluster galaxies, 19 were background galaxies while the remaining 3 were not classified at all. Out of 92 detections, 89 appeared in Ferguson's catalogue and he classified 72 of these as $\mathrm{dE}$ galaxies while the rest were either Spirals, dSO galaxies or appear as groups of galaxies. Caldwell (1987) detected 145 dwarf ellipticals galaxies in the Fornax cluster. Checking against his catalogue, we found that 69 of our APM sample were listed as dE galaxies. Davies et al. (1988) (see also Irwin et al. (1990)) used a similar APM data set with slightly different selection criteria. They obtained a catalogue of 134 LSB galaxies. Checking against this 67 galaxies in our sample were found to have been detected by them.

We have also obtained the redshifts of about 20 galaxies and carried out a numerical simulation to predict the numbers of background galaxies that might be contaminating our sample. In both cases we agree with Ferguson's classification which indicated about 20 background galaxies in our sample. We conclude that this sample consists predominately of Fornax cluster galaxies (full details of the above are given in Morshidi-Esslinger et al., 1999a and b).

The distribution of surface brightnesses for this sample is shown in Fig. 1. The peak occurs at about $23.5 \mathrm{~B} \mu$. This is a result of the high surface brightness cut-off at $22.5 \mathrm{~B} \mu$ and the incompleteness beyond $24 \mathrm{~B} \mu$ and is not indicative 
of the true surface brightness distribution. Due to these selection effects the surface brightness distribution is disjoint from that of the bright galaxy sample.

\section{The VLSB sample}

To obtain data for a sample of lower surface brightness galaxies we have utilized the large format large field of view CCD camera on the Curtis Schmidt Telescope. The $2048 \times 2048$ pixel CCD provides a $1.7 \times 1.7 \mathrm{sq}$ deg field of view with large 2.3 arc sec pixels. The poor response in the blue meant that it was most efficient to use the $R$ filter. We have adjusted all $R$ band surface brightness and magnitudes to the $B$ band calibration of the other samples using $(B-R)=1.5$ (Evans et al. (1990)). We have 12 fields extending outwards from the cluster centre to a distance of $9.5 \mathrm{deg}$. The data have been reduced in the standard way and calibrated against Landolt standards (see Kambas et al., 1999).

We have used the image detection package SExtractor to select a sample of Fornax galaxies. SExtractor detects objects with isophotal areas above a specified size. In this case we used a one sigma isophote that corresponds to $\mu_{L}^{B}=27.5$, almost a factor of 10 fainter than that used for the LSB sample. In the same way as the previous sample we select all objects with exponential scale lengths greater than 3 arc sec, but we set a minimum surface brightness of $\mu_{o}^{B}=24.5$. We have detected 3998 galaxies over the $13.8 \mathrm{sq} \mathrm{deg}$ of the survey. Thus we have a VLSB sample with a surface brightness distribution disjoint from the LSB sample (fig 1). The sample extends down to $M_{B} \approx-10.5$ at the distance of the Fornax custer. This is just about entering the realm of the local group dSph galaxies. We have no way of knowing the contamination of this sample by background galaxies, but we believe that by selecting at such a large scale size we will, in a similar way to the LSB sample, predominantly select nearby galaxies. In the next section we will show that the surface density of galaxies increases rapidly as the cluster centre is approached and so one has strong reasons for believing that in the main these are cluster galaxies. Further details of this work will be given in Kambas et al., (1999).

\section{The radial distribution of galaxies of different surface brightness}

Using the three data sets described above we can measure the decrease in surface number density from the cluster centre, for galaxies of different surface brightnesses, this is shown in fig. 2. Fitting an exponential to the data we find scale sizes of $0.5,1.3$ and $2.0 \mathrm{deg}$ for the bright, LSB and VLSB samples respectively. This corresponds to $0.1,0.3$ and $0.5 \mathrm{Mpc}$ for a Fornax distance of $15 \mathrm{Mpc}$. The LSB and VLSB samples are some 3 and 4 times more spatially extended than the bright galaxy sample. The result for the LSB sample has also been shown to be true using a much larger sample of galaxies over a much larger area of sky that includes other nearby galaxy groups (see Morshidi-Essliger et al. 1999a and b). The central galaxy number densities are 40,10 and 1000 per sq deg respectively. This implies a sharp rise in the luminosity function at the faint end (slope of $\alpha \approx-2$ ) as the VLSB galaxies also have the faintest magnitudes. 


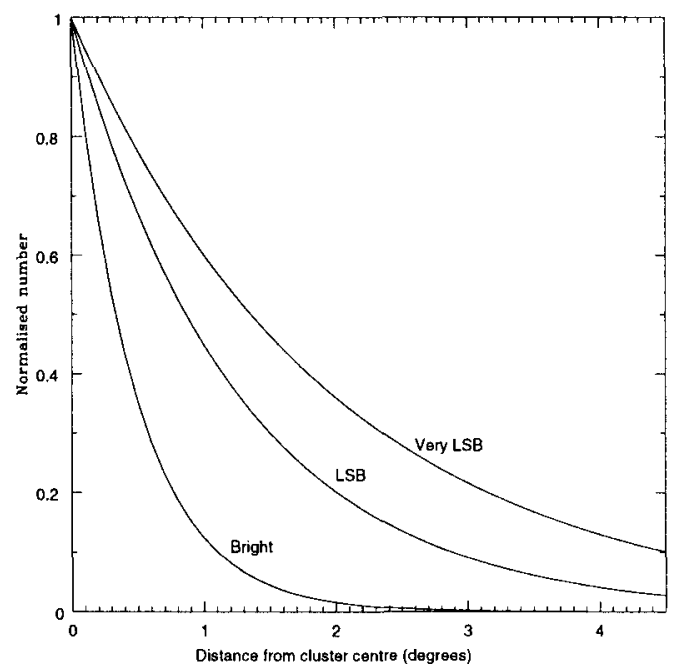

Figure 2. The normalised surface number density of galaxies as a function of distance from the centre of the Fornax cluster.

\section{The total luminosity (mass ?) of each sample}

Given the exponential fits to the number density distributions we can use the mean luminosities of each sample to estimate the ratio of total luminosities produced at each surface brightness. These are 1:0.02:0.5 for the bright:LSB:VLSB samples respectively. The LSB galaxies contribute little to the light of the cluster, but the VLSB galaxies contribute about half that of the brighter galaxies, but this is extended over approximately 16 times the area. The VLSB galaxies also make a VLSB contribution to the total cluster light. We estimate that the intra-cluster light due to the VLSB galaxies is at most $31 \mathrm{~B} \mu$ at the centre of the cluster. The origin of this VLSB population could be the galaxy harrassment mechanism of Moore (this volume). Inter-cluster stars have previously been identified in Fornax by Theuns and Warren (1997) and they state that these stars could account for $40 \%$ of the total cluster light. The mass to light ratios of LSB dwarf galaxies in the local group are large $\left((\mathrm{M} / \mathrm{L})_{\odot}=10-200\right.$ (Irwin and Hatzidimitriou, 1995)), so the VLSB population could contribute the majority of the mass of the cluster and account for the missing mass in clusters.

\section{The extent of the VLSB population}

The Curtis Schmidt data extends to some 9 deg away from the cluster centre and terminates with one field centred on the peculiar lenticular galaxy NGC1291, at a velocity of $802 \mathrm{~km} \mathrm{~s}^{-1}$ (de Vaucouleurs 1975). A visual inspection of the fields around NGC1291 confirms a large number of LSB companions. In fig. 3 we show the normalised surface density of galaxies as a function of distance from 


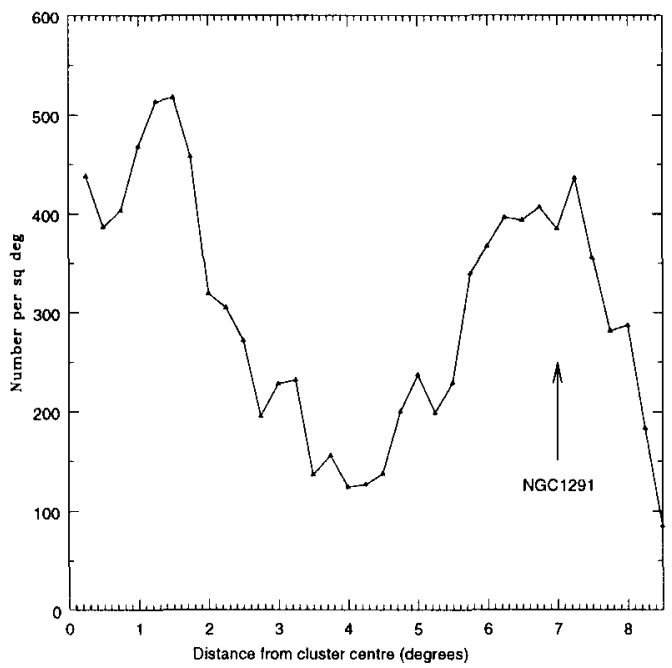

Figure 3. The normalised surface number density of galaxies as a function of distance from the centre of the Fornax cluster with extention to include NGC1291.

the centre of Fornax extending to $9 \mathrm{deg}$. The most striking feature is the initial decline and then large increase in galaxy numbers as NGC1291 is approached. NGC1291 appears to be at the centre of a cluster of LSB galaxies !

\section{Conclusions}

Although our work is at a preliminary stage we believe that it is quite possible that a significant fraction of the mass (but not light) of the Universe resides in small LSB dwarf galaxies that are dark matter dominated. This mass is distributed over scales far larger than that delineated by the bright galaxies.

\section{References}

Abraham R.G., et al., 1996, ApJS, 471, 694

Baugh C.M., Cole S., Frenk C.S., 1996, MNRAS, 283, 1361

Caldwell N., 1983, AJ, 88, 804

Davies J., Phillipps S., Cawson M., disney M. and Kibblewhite E., 1988, MNRAS, 232, 239

de Vaucouleurs G., 1975, AJSS, 284, 29

Disney M., Phillipps S., Davies J., Phillipps S., Cawson M. and Kibblewhite E., 1990, MNRAS, 245, 175

Evans R., Davies J.and Phillipps S., 1990, MNRAS, 245, 164 
Ferguson H.C., 1989, AJ, 98, 367

Irwin M., Davies J., Disney M. and Phillipps S., 1990, MNRAS, 245, 289

Irwin M. and Hadzidimitriou D., MNRAS, 277, 1354

Jenkins et al., 1998, ApJ, 499, 20

Jones J. and Jones B., 1980, MNRAS, 191, 685

Kambas A., Davies J. and Smith R., 1999, MNRAS, in preparation

Maddox S., 1988, $\mathrm{PhD}$ thesis, University of cambridge

Morshidi-Esslinger Z., Davies J. and Smith R., 1999a, MNRAS, in press

Morshidi-Esslinger Z., Davies J. and Smith R., 1999b, MNRAS, in press

Schwartzenberg J., 1996, PhD thesis, University of Bristol

Theuns T. and Warren S., 1997, MNRAS, 284, L11 